\title{
On the manifold of complemented principal inner ideals in $\mathrm{JB}^{*}$-triples.
}

\author{
José M. Isidro * \\ Facultad de Matemáticas, \\ Santiago de Compostela, Spain. \\ jmisidro@zmat.usc.es
}

\author{
László L. Stachó ${ }^{\dagger}$ \\ Bolyai Institute, \\ 6720 Szeged, Hungary. \\ stacho@math.u-szeged.hu
}

December 7, 2005

\begin{abstract}
The set $\mathbb{M}$ of Neher's classes of tripotents in an arbitrary JB*-triple $Z$ is considered and a natural complex-analytic Banach manifold structure is defined on it. The relationship between $\mathbb{M}$ and the Grassmann manifold of all complemented principal inner ideals in $Z$ is studied in detail and the smooth complete vector fields on $\mathbb{M}$ are characterized as smooth complete equivariant vector fields on the manifold $\mathrm{M}$ of tripotents of $Z$.
\end{abstract}

Keywords. JB*-triples, tripotents, principal inner ideals, Grassmann manifolds, complete vector fields.

AMS 2000 Subject Classification. 17C27, 17C36, 17B60, 17B65, $17 \mathrm{~B} 66$.

\section{Introduction}

During the last two decades, great progress has been made in the study of symmetric hermitian complex Banach manifolds of non compact type. One of the results has been the introduction of the category of $\mathrm{JB}^{*}$-triples, which provides a complete axiomatization of those manifolds in Banach algebraic terms, see [9] and [16]. In contrast, their duals, the complex symmetric hermitian manifolds of compact type, have received almost no attention, see [4], [12], [13]. Recently, Kaup [10] has described an interesting example of these dual Banach manifolds: the family $\mathbb{P}$ of all complemented principal inner ideals of a $\mathrm{JB}^{*}$-triple $Z$ as a submanifold of the Grassmannian of all complemented subspaces of $Z$. In the construction of $\mathbb{P}$ the $\operatorname{set} \operatorname{Reg}(Z)$ of von Neumann regular elements of $Z$ and the set $\mathrm{M}$ of non zero tripotents of $Z$ play a decisive role. Indeed, a principal inner ideal $J$ of $Z$ is complemented if and only if it is generated by an element of $\operatorname{Reg}(Z)$, in which case it is also generated by a tripotent of $Z$. Yet different tripotents

\footnotetext{
*Supported by Ministerio de Educación y Cultura of Spain, Research Project BFM2002-01529.

${ }^{\dagger}$ Supported by the Bilateral Spanish-Hungarian Project E-50/2002 and Hungarian Research Grant OTKA T34267
} 
$e, f \in \mathrm{M}$ may give rise to the same principal inner ideal, which occurs if and only if they are equivalent in the sense of Neher (for details and definitions, see below).

Our aim in this paper is to study the holomorphic structure of $\mathbb{P}$ in terms of tripotents. Notice that points in an open set $\mathbf{U}$ in $\mathbb{P}$ are subspaces of $Z$ that in general may have large intersections. Hence the task of representing holomorphic maps on $\mathbf{U}$ in a canonical manner by holomorphic maps on an open subset $U$ of $Z$ is by no means a trivial task. We start from the observation that distinct principal inner ideals have disjoint intersections with $\mathrm{M}$, and that the family $\mathbb{M}=\{J \cap \mathrm{M}: J \in \mathbb{P}\}$ consists of all Neher's equivalence classes of tripotents. Recall that two tripotents $e, f \in Z$ are equivalent ( $e \sim f$ in notation) in the sense of Neher if they have the same box operator $D(e)=D(f)$. Hence it is also possible to represent $\mathbb{P}$ either as the family of inner triple derivations $\mathbb{D}:=\{i D(e): e$ a tripotent $\}$ or as the quotient set $\mathbb{M}:=\mathrm{M} / \sim$ of classes of equivalence. Thus we have alternative convenient possibilities to study the topology, the local complex structure and the global complete holomorphic vector fields on $\mathbb{P}$ by means of the commutative diagram

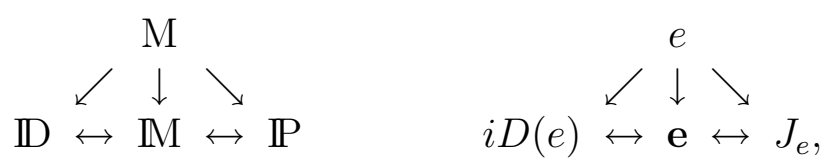

where $\mathbf{e}:=\pi(e)$ stands for the equivalence class of $e \in \mathrm{M}$ and $\pi: \mathrm{M} \rightarrow \mathbb{M}$ denotes the canonical map. In particular we can regard $M$ as a fibre manifold over $\mathbb{P}$. As one of our main results we prove that a mapping $\Phi: \mathbf{U} \rightarrow Y$ from an open subset $\mathbf{U}$ of $\mathbb{I}$ into a Banach space $Y$ is holomorphic if and only if for any tripotent $e \in \pi^{-1}(\mathbf{U})$ there is a holomorphic function $\varphi_{e}: U_{e} \rightarrow Y$ defined in some neighborhood $U_{e}$ of $e$ in $Z$ such that $\varphi_{e}(f)=\Phi(\mathbf{f})$ whenever $f \in \mathrm{M} \cap U_{e}$ and $\pi(f)=\mathbf{f}$. Before getting that result we we study the natural real manifold structure of $\mathbb{P}$ via the above diagram. We give a detailed description of the topology of $\mathbb{P}$ in terms of the Hausdorff distance that $\mathbb{M}$ inherits from $Z$ and, alternatively in terms of the operator distance on $\mathbb{D}$. As a main tool, we stablish that Lie algebra of smooth vector fields on $\mathbb{P}$ is isomorphic to a Lie subalgebra of smooth -equivariant vector fields on $\mathrm{M}$ that we characterize in terms of the Peirce projectors of $Z$. We prove that if $Z$ is a $\mathrm{JC}^{*}$-triple then there is a canonical holomorphic atlas for $\mathbb{P}$ of the form $\left\{T_{e}: e \in \mathrm{M}\right\}$ where $T_{e}(u)=s(\exp D(e, u) e)$ $\left(u \in Z_{1 / 2}(e)\right)$ and $s(x)$ stands for the support tripotent of $x$. We conjecture that this result can be extended by Shirsov-Cohn type arguments to general JB*-triples.

For a study of some these topics in the finite-dimensional setting see [11] chapter 5. However, our methods are not those of Loos due to the lack of local compactness.

\section{Preliminaries}

Throughout the whole work we deal with complex structures without mentioning it later. JB*triples are Banach spaces with holomorphically symmetric unit ball. It is known that they are those Banach spaces that can be endowed with a necessarily unique triple product $\{x y z\}$ (an operation $Z \times Z \times Z \rightarrow Z$ ) that is symmetric bilinear in the variables $x, z$ and conjugate linear in $y$, satisfies the $C^{*}$-axiom $\|\{x x x\}\|=\|x\|^{3}$ and such that, by writing $D(a, b)$ for the polarized derivation

$$
D(a, b): x \mapsto\{a b x\}, \quad D(a):=D(a, a),
$$


the operators $i D(a)$ are derivations of the triple product $\{\cdots\}$ and each $D(a)$ is positive hermitian with respect to the norm $\|$.$\| , i.e.$

$$
\begin{aligned}
& i D(a)\{x y z\}=\{[i D(a) x] y z\}+\{x[i D(a) y] z\}+\{x y[i D(a) z]\} \\
& \text { and }\|\exp \zeta D(a)\| \leq 1 \text { for } \zeta \in \mathbb{C} \text { with } \Re \zeta \leq 0 .
\end{aligned}
$$

As a typical example, $\mathrm{C}^{*}$-algebras are $\mathrm{JB}^{*}$-triples with the triple product $\{x y z\}:=\left(x y^{*} z+\right.$ $\left.z y^{*} x\right) / 2$. An automorphism of $Z$ is a linear map $\lambda: Z \rightarrow Z$ such that $\lambda\{x y z\}=\{(\lambda x)(\lambda y)(\lambda z)\}$, $(x, y, z \in Z)$, in which case $\lambda$ necessarily is a bounded operator. A derivation of $Z$ is a linear $\operatorname{map} \delta: Z \rightarrow Z$ such that $\delta\{x y z\}=\{(\delta x) y z\}+\{x(\delta y) z\}+\{x y(\delta z)\},(x, y, z \in Z)$, in which case $\delta$ is necessarily a bounded operator. Recall [16] that the set $\operatorname{Aut}(Z)$ of automorphisms of $Z$ is in a natural way a real Banach-Lie group whose Banach-Lie algebra is $\mathfrak{D}:=\operatorname{Der}(Z)$, the space of all derivations of $Z$.

Henceforth $Z$ stands for an arbitrarily fixed $\mathrm{JB}^{*}$-triple with triple product $\{\cdots\}$. A particular role is played by the tripotents in $\mathrm{JB}^{*}$-triple theory. They are the elements with the projection property $e=\{e e e\}$. We write $\mathrm{M}:=\mathrm{M}(Z)$ for the set of all non-zero tripotents in $Z$. A $\mathrm{JB}^{*}$-triple may have no non-zero tripotents but it has plenty of them if $Z$ is a dual Banach space. In the case of the $\mathrm{C}^{*}$-algebra $\mathcal{L}(H)$ where $H$ is a Hilbert space, tripotents are exactly partial isometries. For each tripotent $e$, the derivation $i D(e)$ is a simple algebraic operator with spectrum in $\{0, i / 2, i\}$, that is

$$
D(e)=\sum_{k=0}^{2} \frac{k}{2} P_{k / 2}(e),
$$

where

$$
P_{k / 2}(e): Z \rightarrow Z_{k / 2}(e):=\{z \in Z: D(e) z=(k / 2) z\}, \quad P_{k / 2}(e) P_{\ell / 2}(e)=\delta_{k, \ell} P_{k / 2}(e) .
$$

The projections $P_{k / 2}(e)$ are called the Peirce projections of $e$ and the spectral subspaces $Z_{k / 2}(e)$ are the Peirce spaces of $e$. Since $i D(e)$ is a triple derivation, we have the Peirce arithmetic rules

$$
\begin{aligned}
& \left\{Z_{k / 2}(e) Z_{\ell / 2}(e) Z_{m / 2}(e)\right\} \subset Z_{(k-\ell+m) / 2}(e) \\
& D\left(Z_{0}(e), Z_{1}(e)\right)=\{0\}=D\left(Z_{1}(e), Z_{0}(e)\right),
\end{aligned}
$$

where $Z_{\xi}(e)=\{0\}$ if $\xi \notin\{0,1,2\}$. For $a \in Z$, a conjugate-linear quadratic representation operator $Q(a) \in \mathcal{L}(Z)$ is defined by $Q(a) z:=\{a z a\}$ for $z \in Z$. We note that $Q(e)^{2}=P_{1}(e)$ and $Q(e)^{3}=Q(e)$ for any $e \in \mathrm{M}$. As a consequence we have

$$
Z_{1}(e)=A(e) \oplus i A(e) \quad P_{1}(e)=P_{1}^{+}(e)+P_{1}^{-}(e),
$$

where $A(e):=\left\{z \in Z_{1}(e): Q(e) z=z\right\}$ and $P^{ \pm}(e):=\frac{1}{2}\left(P_{1}(e) \pm Q(e)\right)$ are the projections from $Z$ onto $A(e)$ and $i A(e)$ respectively. We shall also use the direct sum decomposition

$$
Z=Z^{+}(e) \oplus Z^{-}(e):=\left[A(e) \oplus Z_{0}(e)\right] \oplus\left[i A(e) \oplus Z_{1 / 2}(e)\right]
$$

where notation is selfexplanatory, and denote by $F^{+}(e)$ and $F^{-}(e)$ the projectors from $Z$ onto $Z^{+}(e)$ and $Z^{-}(e)$ respectively.

For each $w \in Z$, the set $J_{w}:=Q(w) Z=\{w Z w\}$ satisfies $\left\{J_{w} Z J_{w}\right\} \subset J_{w}$ and is a closed subspace of $Z$ called the principal inner ideal generated by $w$. An element $w \in Z$ is said to be 
von Neumann regular if there exists a tripotent $e \in \mathrm{M}$ that generates the same principal inner ideal as $w$, that is, if $\{w Z w\}=\{e Z e\}$. For instance, an operator $a \in \mathcal{L}(H)$ is von Neumann regular in $Z=\mathcal{L}(H)$ if and only if its restriction to $\operatorname{ker}(a)^{\perp}$ is bounded from below. In that case $\{a \mathcal{L}(H) a\}=a \mathcal{L}(H) a=e \mathcal{L}(H) e$ with any partial isometry $e$ such that $\operatorname{ran}(e)=\operatorname{ran}(a)$ and $\operatorname{ker}(e)=\operatorname{ker}(a)$. We know [10] that $J_{w}$ is a complemented subspace in $Z$ if and only if $w$ is von Neumann regular. Yet different tripotents $e$ and $f$ may give rise to the same principal inner ideal. This occurs if and only if $D(e)=D(f)$ or simply if $e \in Z_{1}(f)$ and $f \in Z_{1}(e)$ in which case $e$ and $f$ are said to be equivalent in the sense of Neher and we write $e \sim f$, ([14] Theorem 2.3). ${ }^{1}$ We write $\mathbb{P}$ for the set of all complemented principal inner ideals in $Z$ considered as a submanifold of the Grassmannian manifold associated with $Z$. For our purposes it suffices to note that $\mathbb{P}:=\left\{J_{e}: e \in \mathrm{M}\right\}$ and that $\mathcal{X}:=\left\{X_{e}: e \in \mathrm{M}\right\}$ is a holomorphic atlas for $\mathbb{P}$, where

$$
X_{e}: u \mapsto(\exp D(u, e)) J_{e} \quad\left(u \in Z_{1 / 2}(e)\right)
$$

Recall that a subset $S$ of a real Banach space $B$ is a direct submanifold of $B$ if for every point $p \in S$ there exists a neighborhood $U$ of $p$ in $B$ along with a direct decomposition $B=B_{1} \oplus B_{2}$ and a smoothly invertible one-to-one map $\Phi: V \rightarrow U$, where $V$ is some neighborhood of the origin in $B$, such that $S \cap U=\Phi\left(B_{1} \cap V\right)$. We then say that $B_{1}$ is the tangent space to $S$ at the point $p$ and that $(U, V, \Phi)$ is a local chart for $S$ at $p$. It is customary to identify the tangent space $B_{1}$ with its isomorphic image under $\Phi^{\prime}(0)$, that is, the space consisting of the tangent vectors to smooth curves starting from $p$ and ranging in $S$,

$$
\mathrm{T}_{p} S=\left\{w \in B: \exists x: \mathbb{R} \rightarrow S \text { with } w=\left.\frac{d}{d t}\right|_{t=0} x(t)\right\}=\Phi^{\prime}(0) B_{1} .
$$

We denote by $\mathrm{T} S:=\left\{(p, w): p \in S, w \in \mathrm{T}_{p} S\right\}$ the tangent bundle to $S$. Given a direct submanifold $S$ of $B$, if $W: S \rightarrow B$ is a locally Lipschitzian mapping such that $W(p) \in \mathrm{T}_{p} S$ for every point $p \in S$, then there is a unique curve $x_{p}: I_{p} \rightarrow S$ (where $I_{p}$ is the maximal open interval around 0 in $\mathbb{R}$ ) such that $x_{p}(0)=p$ and $\frac{d}{d t} x_{p}(t)=W\left(x_{p}(t)\right),\left(t \in I_{p}\right)$. For fixed $t \in \mathbb{R}$, the mapping $p \mapsto x_{p}(t)$ is denoted by $\exp t W$ and called the exponential of the vector field $t W$. Notice that $\exp t W:\left\{p \in S: t \in I_{p}\right\} \rightarrow S$. For instance, $\mathrm{M}$ is a real-analytic direct submanifold of $Z$ and $\mathrm{T}_{e} \mathrm{M}=Z^{-}(e)$ for all $e \in \mathrm{M}$, see [3].

For details on $\mathrm{JB}^{*}$-triples and Banach manifolds see [11] and [16].

\section{Real manifold structures on $\mathrm{D}$ and $\mathrm{M}$}

We begin with a topological study of the family $\mathbb{D}$. This requires a detailed analysis of the inner derivations $K(e, v),\left(e \in M, v=u+i a \in Z^{-}(e)\right)$, defined by

$$
K(e, u+i a):=2(D(u, e)-D(e, u))+\frac{i}{2}(D(a, e)+D(e, a))
$$

for which we need some notation and technical results. Given a tripotent $e \in \mathrm{M}$ we define

$$
\pi_{k \ell}(e) L:=P_{k / 2}(e) L P_{\ell / 2}(e), \quad \Pi_{m}(e):=\sum_{|k-\ell|=m} \pi_{k \ell}(e) \quad(L \in \mathcal{L}(Z))
$$

\footnotetext{
${ }^{1}$ The proof of $Z_{1}(e)=Z_{1}(f) \Rightarrow D(e)=D(f)$ in [14] Theorem 2.3 page 18 contains a gap, though the result is true.
} 
Notice that the operators $\pi_{k \ell}(e)$ are pairwise orthogonal projections (in Banach space sense) on $\mathcal{L}(Z)$ and $\mathcal{L}(Z)=\oplus_{k, \ell=0}^{2} \pi_{k \ell}(e) \mathcal{L}(Z)$. Furthermore, since $D(e)=\sum_{k=0}^{2} \frac{k}{2} P_{k / 2}(e)$, the Lie adjoint of $D(e)$ is the map $D(e)_{\#}: L \mapsto[D(e), L]=\sum_{k, \ell=0}^{2} \frac{k-\ell}{2} \pi_{k \ell}(e) L$, hence

$$
D(e)_{\#}^{2}=\sum_{k, l=0}^{2}\left(\frac{k-l}{2}\right)^{2} \pi_{k, l}(e)=\sum_{m=0}^{2}\left(\frac{m}{2}\right)^{2} \Pi_{m}(e) .
$$

In particular the projections $\Pi_{m}(e)$ are real polynomials of $D(e)_{\#}^{2}$. Since $i D(e)_{\#}$ maps $\mathfrak{D}$ into itself, it follows that each operator $\Pi_{m}(e)$ is a projection of $\mathfrak{D}$ onto $\left\{L \in \mathfrak{D}: D(e)_{\#}^{2} L=\right.$ $\left.(m / 2)^{2} L\right\}$ and $\mathfrak{D}=\oplus_{m=0}^{2} \mathfrak{D}_{m}(e)$ where $\mathfrak{D}_{m}(e):=\Pi_{m}(e) \mathfrak{D}$.

3.1. Lemma. The map $\phi: u \mapsto K(e, u)$ is a real-linear Banach space isomorphism $Z_{1 / 2}(e) \leftrightarrow$ $\mathfrak{D}_{1}(e)$. Moreover $\mathfrak{D}_{2}(e)=\{0\}$

Proof. First we claim that for any derivation $L$ such that $L e \in Z_{1 / 2}(e)$, we have

$$
D(e)_{\#}^{2} L=\frac{1}{4} K(e, L e) \quad\left(L \in \mathfrak{D} \text { with } L e \in Z_{1 / 2}(e)\right) .
$$

Indeed, any derivation $L$ satisfies

$$
L\{e e x\}=\{(L e) e x\}+\{e(L e) x\}+\{e e(L x)\} \quad(x \in Z),
$$

in particular if $L e \in Z_{1 / 2}(e)$ then

$$
D(e)_{\#} L=-(D(L e, e)+D(e, L e))=\frac{i}{2} K(e, i L e) \in i K(e, Z) \subset \mathrm{i} \mathfrak{D} .
$$

Applying (4) to $\tilde{L}:=i D(e)_{\#} L=-\frac{1}{2} K(e, i L e)$ which satisfies $\tilde{L} e=-\frac{i}{2} L e \in Z_{1 / 2}(e)$, we get

$$
i D(e)_{\#}^{2} L=D(e)_{\#}\left(i D(e)_{\#} L\right)=D(e)_{\#} \tilde{L}=\frac{i}{2} K(e, i \tilde{L} e)=\frac{i}{4} K(e, L e)
$$

whence (3) holds. Now we prove the lemma.

i). Clearly $K(e, u) \in \mathfrak{D}$ for all $u \in Z_{1 / 2}(e)$. Moreover for $L=\phi(u)=K(e, u)$ with $u \in Z_{1 / 2}(e)$ we have $L e \in Z_{1 / 2}(e)$ and so (3) is valid, hence $4 D(e)_{\#}^{2} L=K(e, K(e, u) e)=$ $K(e, u)=L$ and therefore $\phi(u) \in \mathfrak{D}_{1}(e)$.

Assume now that $L$ satisfies $L \in \mathfrak{D}_{1}(e)$. Then $L=4 \Pi_{1}(e) L=\sum_{|k-l|=1} \pi_{k, l} L$ from which we get $L\left(Z_{1}(e)\right) \subset Z_{1 / 2}(e)$ and so (3) holds. Also from $L \in \mathfrak{D}_{1}(e)$ we get $4 D(e)_{\#}^{2} L=L$, and finally $L=4 D(e)_{\#}^{2} L=K(e, L e)=K(e, u)$ where $u=L e \in Z_{1 / 2}(e)$. The remainder is obvious since $\phi$ is real-linear and injective.

ii). Assume now $L \in \mathfrak{D}_{2}(e)$ that is $L=\Pi_{2}(e) L=\sum_{|k-\ell|=2} \pi_{k \ell} L=\left(\pi_{02}(e)+\pi_{20}(e)\right) L$ and so $L e=0$. On the other hand $L \in \mathfrak{D}_{2}(e)$ combined with (3) yields $L=D(e)_{\#}^{2} L=$ $\frac{1}{4} K(e, L e)=0$.

We are now ready for one of the main results of this section.

3.2. Theorem. Let $Z$ and $M$ denote respectively an arbitrary $J B^{*}$-triple and the set of its non zero tripotents. Then $\mathbb{D}:=\{i D(e): e \in \mathrm{M}\}$ is a real-analytic direct submanifold of $\mathfrak{D}$ with tangent space at the point $\mathrm{iD}(\mathrm{e})$ given by $\mathrm{T}_{\mathrm{iD}(\mathrm{e})} \mathrm{D}=\left\{K(e, u): u \in Z_{1 / 2}(e)\right\}$. 
Proof. Let $e \in \mathrm{M}$ be fixed. Recall that $\mathfrak{D}=\mathfrak{D}_{1}(e) \oplus \mathfrak{D}_{0}(e)$ where

$$
\mathfrak{D}_{1}(e)=\left\{K(e, u): u \in Z_{1 / 2}(e)\right\}
$$

by (3.1). In terms of this decomposition, consider the real analytic mapping $\Psi_{e}: \mathfrak{D} \rightarrow \mathfrak{D}$ defined by

$$
\Psi_{e}: K(e, u) \oplus L \mapsto(\exp K(e, u))(i D(e)+L)(\exp K(e, u))^{-1} .
$$

For the Fréchet derivative of $\Psi_{e}$ at the origin we have

$$
\Psi_{e}^{\prime}(0,0)=\left(\frac{\partial \Psi_{e}}{\partial K}, \frac{\partial \Psi_{e}}{\partial L}\right)_{\left.\right|_{(0,0)}}=\left(-i D(e)_{\#}, \text { Id }\right)
$$

From (4) applied to $L=K(e, u)$ we get $D(e)_{\#} K(e, u)=\frac{i}{2} K(e, i u)$ hence $i D(e)_{\#}$ preserves the space $\mathfrak{D}_{1}(e)$, and moreover $i D(e)_{\#} \mid \mathfrak{D}_{1}(e)$ is invertible and so is $\Psi_{e}^{\prime}(0,0)$. Thus, by the Inverse Function Theorem, $\Psi_{e}$ is real-bianalytic on some neighborhood $U$ of $(0,0)$, say $\Psi_{e}: U \leftrightarrow V$, where $V$ is some neighborhood of $i D(e)$ in $\mathfrak{D}$. On the other hand, a derivation $K(e, u)+L$ with $u \in Z_{1 / 2}(e)$ and $L \in \mathfrak{D}_{0}(e)$ belongs to $\mathfrak{D}_{1}(e)$ if and only if $L=0$. Since the linear operators $\exp K(e, u)$ are automorphisms of $Z$, it follows that $V \cap \mathbb{D}=\Psi_{e}\left(U \cap \mathfrak{D}_{1}(e)\right)$. That is, $\mathbb{D}$ is a direct submanifold of $\mathfrak{D}(Z)$. Therefore

$$
\begin{aligned}
\mathrm{T}_{\mathrm{iD}(\mathrm{e})} \mathbb{D}=\Psi^{\prime}(0,0) \mathfrak{D}_{1}(e) & =-i D(e)_{\#}\left\{K(e, u): u \in Z_{1 / 2}(e)\right\}= \\
\left\{K(e, i u): u \in Z_{1 / 2}(e)\right\} & =\left\{K(e, u): u \in Z_{1 / 2}(e)\right\} .
\end{aligned}
$$

This completes the proof of the theorem.

3.3. Corollary. The family of maps $\widehat{\mathcal{X}}:=\left\{\widehat{X}_{e}: e \in \mathrm{M}\right\}$ where

$$
\widehat{X}_{e}: u \mapsto i D([\exp K(e, u)] e), \quad u \in Z_{1 / 2}(e)
$$

is a real-analytic atlas for $\mathbb{D}$.

Transferring the manifold structure of $\mathbb{D}$ to $\mathbb{M}$ by the bijection $i D(e) \leftrightarrow$ e we get

3.4. Corollary. The family of maps $\widetilde{\mathcal{X}}:=\left\{\widetilde{X}_{e}: e \in \mathrm{M}\right\}$ where

$$
\widetilde{X}_{e}: u \mapsto\left\{f \in \mathrm{M}: i D(f)=\widehat{X}_{e}(u)\right\}=\pi([\exp K(e, u)] e), \quad u \in Z_{1 / 2}(e)
$$

is a real-analytic atlas for $\mathbb{M}$.

Thus by construction $\mathbb{D}$ and $\mathbb{M}$ are isomorphic as manifolds, however a priori is not clear whether $\mathbb{D}$ and $\mathbb{M}$ are isomorphic to $\mathbb{P}$ as defined by Kaup in [10], as we shall see later on. Since $\mathbb{D}$ a direct submanifold in $\mathfrak{D}$, the topology defined on $\mathbb{D}$ by the atlas $\widehat{\mathcal{X}}$, with basis of open sets $\left\{\widehat{U}_{e, \delta}: e \in \mathrm{M}, \delta>0\right\}$ where $\widehat{U}_{e, \delta}:=\left\{\widehat{X}_{e}(u): u \in Z_{1 / 2}(e),\|u\|<\delta\right\}$, coincides with the topology inherited from $\mathfrak{D}$. Now we study the topology on $\mathrm{M}$ defined by the atlas $\tilde{\mathcal{X}}$

3.5. Proposition. Let e, $f \in \mathrm{M}$ satisfy $\|D(e)-D(f)\|<1 / 66$. Then there exists $e^{\prime \prime} \in \mathrm{M}$ such that $e^{\prime \prime} \sim f$ and $\left\|e-e^{\prime \prime}\right\| \leq 16\|D(e)-D(f)\|$. 
Proof. For any $a, b \in Z$ we have $D(a)-D(b)=D(a, a-b)+D(a-b, b)$. Hence

$$
\begin{gathered}
\|D(a)-D(b)\| \leq(\|a\|+\|b\|)\|a-b\| \\
D(a)^{2}-D(b)^{2}=D(a)[D(a)-D(b)]+[D(a)-D(b)] D(b) \\
\left\|D(a)^{2}-D(b)^{2}\right\| \leq(\|D(a)\|+\|D(b)\|)\|D(a)-D(b)\| \leq \\
\leq\left(\|a\|^{2}+\|b\|^{2}\right)\|D(a)-D(b)\| .
\end{gathered}
$$

Now we prove the proposition. Let $e, f \in \mathrm{M}$ satisfy $\|D(e)-D(f)\| \leq \varepsilon$ for some $\varepsilon>0$. Define $e^{\prime}:=P_{1}(f) e$. Since $\left\|e^{\prime}\right\| \leq\|e\|=\|D(e)\|=\|D(f)\|=1$, the above inequalities and $P_{1}(f) e=\left(2 D(f)^{2}-D(f)\right) e$ yield

$$
\begin{gathered}
\left\|e^{\prime}-e\right\|=\left\|2\left[D(f)^{2}-D(e)^{2}\right] e+[D(e)-D(f)] e\right\| \leq \\
\leq 2\left(\|e\|^{2}+\|f\|^{2}\right)\|D(e)-D(f)\|+\|D(e)-D(f)\| \leq 5 \varepsilon,
\end{gathered}
$$

therefore

$$
\left\|D\left(e^{\prime}\right)-D(e)\right\| \leq\left(\left\|e^{\prime}\right\|+\|e\|\right)\left\|e^{\prime}-e\right\| \leq 10 \varepsilon .
$$

In particular, if $\varepsilon<1 / 11$ then

$$
\begin{gathered}
\left\|\operatorname{id}_{Z_{1}(f)}-\left.D\left(e^{\prime}\right)\right|_{Z_{1}(f)}\right\|=\left\|\left.\left(D(f)-D\left(e^{\prime}\right)\right)\right|_{Z_{1}(f)}\right\| \leq \\
\leq\|D(f)-D(e)\|+\left\|\left.\left(D(e)-D\left(e^{\prime}\right)\right)\right|_{Z_{1}(f)}\right\| \leq 11 \varepsilon<1 .
\end{gathered}
$$

Thus the operator $\left.D\left(e^{\prime}\right)\right|_{Z_{1}(f)}$, which maps the subtriple $Z_{1}(f)$ into itself, is invertible and its spectrum satisfies

$$
\operatorname{Sp}\left(\left.D\left(e^{\prime}\right)\right|_{Z_{1}(f)}\right) \geq 1-11 \varepsilon
$$

whenever $\varepsilon<1 / 11$. Henceforth assume $\varepsilon<1 / 11$. Then by the odd functional calculus it follows that there exists a linear isometry $T: \mathcal{C}(\Omega) \rightarrow Z_{1}(f)$, where $\Omega:=\left.\operatorname{Sp} D\left(e^{\prime}\right)\right|_{Z_{1}(f)} \geq 0$, such that $T\left(\operatorname{id}_{\Omega}\right)=e^{\prime}$ and $T(\varphi \bar{\psi} \theta)=\{T(\varphi) T(\psi) T(\theta)\}$ for all functions $\varphi, \psi, \theta \in \mathcal{C}(\Omega)$. Consider the element $e^{\prime \prime}:=T(1)$ where 1 is the constant unit function on $\Omega$. Then $e^{\prime \prime}$ is a tripotent in $Z_{1}(f)$ and we have $\left\|e^{\prime}-e^{\prime \prime}\right\|=1-\min \Omega \leq 11 \varepsilon$. It follows

$$
\begin{gathered}
\left\|D\left(e^{\prime \prime}\right)-D(f)\right\| \leq\left\|D\left(e^{\prime \prime}\right)-D\left(e^{\prime}\right)\right\|+\left\|D\left(e^{\prime}\right)-D(e)\right\|+\|D(e)-D(f)\| \leq \\
\leq\left(\left\|e^{\prime \prime}\right\|+\left\|e^{\prime}\right\|\right)\left\|e^{\prime \prime}-e^{\prime}\right\|+\left(\left\|e^{\prime}\right\|+\|e\|\right)\left\|e^{\prime}-e\right\|+\|D(e)-D(f)\| \leq 33 \varepsilon
\end{gathered}
$$

Thus if $33 \varepsilon<1 / 2$, that is if $\varepsilon<1 / 66$, then $\operatorname{Sp}\left(\left.D\left(e^{\prime \prime}\right)\right|_{Z_{1}(f)}\right) \geq 1-33 \varepsilon>1 / 2$. However, since $e^{\prime \prime}$ is a tripotent, we must have $\operatorname{Sp} D\left(e^{\prime \prime}\right) \subset\{0,1 / 2,1\}$. Therefore

$$
\left.D\left(e^{\prime \prime}\right)\right|_{Z_{1}(f)}=\left.D(f)\right|_{Z_{1}(f)}=\operatorname{Id}_{Z_{1}(f)} \quad \text { if } \quad \varepsilon<1 / 66 .
$$

In particular $\left\{e^{\prime \prime} e^{\prime \prime} f\right\}=f$ and $\left\{f f e^{\prime \prime}\right\}=e^{\prime \prime}$ which proves $e^{\prime \prime} \sim f$. Moreover $\left\|e^{\prime \prime}-e\right\| \leq$ $\left\|e^{\prime}-e\right\|+\left\|e^{\prime \prime}-e^{\prime}\right\| \leq 16 \varepsilon$ if $\varepsilon<1 / 66$.

Recall that the Hausdorff distance $d$ in $\mathbb{M}$ is defined by

$$
d(\mathbf{e}, \mathbf{f}):=\max \left\{\sup _{e \in \mathbf{e}} \inf _{f \in \mathbf{f}}\|e-f\|, \sup _{f \in \mathbf{f}} \inf _{e \in \mathbf{e}}\|e-f\|\right\} .
$$


In course of the proof we have seen that $d(\mathbf{e}, \mathbf{f}) \leq 16\|D(e)-D(f)\| \leq 32\|e-f\|$ whenever $\mathbf{e}, \mathbf{f} \in \mathbb{M}$ and $\|D(e)-D(f)\| \leq 1 / 66$. On the other hand, given $\mathbf{e}, \mathbf{f} \in \mathbb{M}$ there are $e \in \mathbf{e}$ and $f \in \mathbf{f}$ such that $\|e-f\| \leq 2 d(\mathbf{e}, \mathbf{f})$ hence

$$
\|D(e)-D(f)\| \leq 2\|e-f\| \leq 4 d(\mathbf{e}, \mathbf{f}) .
$$

3.6. Corollary. i) The topology defined on $\mathrm{M}$ by the Hausdorff distance of $\mathrm{M} / \sim$ coincides with that defined by the bias $\delta(\mathbf{e}, \mathbf{f}):=\inf _{e \in \mathbf{e}, f \in \mathbf{f}}\|e-f\|$.

ii) The mapping $\mathbf{e} \mapsto i D(e)$ is bilipschitzian between the space $\mathbb{M}$ equipped with the Hausdorff distance and the operator family $\mathrm{D}$ equipped with the distance of the operator norm.

iii) The topology defined by the Hausdorff distance on $\mathbb{M}$ coincides with the canonical quotient topology.

\section{An auxiliary manifold}

The manifolds $\mathrm{M}$ and $\mathrm{P}$ have been studied separately in [3] and [10]. To study their relationships we introduce the auxiliary manifold

$$
\mathrm{S}:=\bigcup_{e \in \mathrm{M}}\{e\} \times Z_{1}(e)=\left\{(e, x): e \in \mathrm{M}, x \in Z_{1}(e)\right\} \subset Z \times Z .
$$

It might be helpful to visualize $\mathrm{S}$ as the subset of $Z \times Z$ obtained by attaching to each point $e \in \mathrm{M}$ the inner ideal $J_{e}=Z_{1}(e)$ that it generates. We also consider tangent vectors and tangent vector fields to $\mathrm{S}$.

4.1. Theorem. Let $Z, M$ and $\mathrm{S}$ denote respectively an arbitrary JB*-triple, the set of its non zero tripotents and the subset of $Z \times Z$ defined in (5). Then $\mathrm{S}$ is a real-analytic direct submanifold of $Z \times Z$ whose tangent space at $(e, x) \in \mathrm{S}$ is $\mathrm{T}_{(e, x)}(\mathrm{S})=Z^{-}(e) \times Z_{1}(e)$.

Proof. Clearly $Z^{-}(e) \times Z_{1}(e)$ is a direct summand in $Z \times Z$ and given $(e, x) \in \mathrm{S}$, the function $\Phi_{(e, x)}: Z \times Z \rightarrow Z \times Z$ defined by

$$
\Phi_{(e, x)}(w, z):=\left(\left[\exp K\left(e, F^{-}(e) w\right)\right]\left[e+F^{+}(e) w\right],\left[\exp K\left(e, F^{-}(e) w\right)\right](x+z)\right)
$$

is real-analytic with $\Phi_{(e, x)}(0,0)=(e, x)$ and its Fréchet derivative at $(0,0)$ is the operator

$$
\Phi_{(e, x)}^{\prime}(0,0):=(h, k) \mapsto\left(h, K\left(e, F^{-}(e) h\right) x+k\right) \quad(h, k) \in Z \times Z
$$

which is invertible. We claim that, for $(w, z)$ in a neighbourhood of $(0,0) \in Z \times Z$, we have

$$
\Phi_{(e, x)}(w, z) \in \mathrm{S} \Longleftrightarrow\left(w \in Z^{-}(e) \text { and } z \in Z_{1}(e)\right)
$$

An application of the inverse mapping theorem will then give that $\mathrm{S}$ is a direct submanifold of $Z \times Z$, that the tangent space $\mathrm{T}_{(e, x)}$ to $\mathrm{S}$ at $(e, x)$ is $Z^{-}(e) \times Z_{1}(e)$ or its isomorphic image under $\Phi_{(e, x)}^{\prime}(0,0)$, and that a local chart at $(e, x)$ is $(w, z) \mapsto \Phi_{(e, x)}(w, z)$ where $(w, z)$ ranges in a neighbourhood of $(0,0)$ in $Z^{-}(e) \times Z_{1}(e)$. 
Set $g_{w}:=\exp K\left(e, F^{-}(e) w\right)$ for $w \in Z^{-}(e)$. Then $g_{w} \in \operatorname{Aut}(Z)$ hence $g_{w}(e) \in \mathrm{M}$. We project the relation " $\Phi_{(e, x)} \in \mathrm{S}$ " onto the factor spaces of $Z \times Z$. By the definition of S, these projections are

$$
\begin{aligned}
& {\left[\exp K\left(e, F^{-}(e) w\right)\right]\left(e+F^{+}(e) w\right) \in \mathrm{M},} \\
& g_{w}(x+z) \in Z_{1}\left(g_{w}(e)\right)=g_{w}\left(Z_{1}(e)\right) .
\end{aligned}
$$

Notice that (7) does not involve the coordinate $z$. From the description of the manifold M (see Sauter's Ph. D., Satz 4.4) we know that, in a neighbourhood of $0 \in Z$, (7) is equivalent to $w \in Z^{-}(e)=i A(e) \oplus Z_{1 / 2}(e)$. Applying $g_{w}^{-1}$ to (8) this relation becomes $x+z \in Z_{1}(e)$. Remark that $x \in Z_{1}(e)$ since $(e, x) \in \mathrm{S}$, hence the latter is equivalent to $z \in Z_{1}(e)$.

The following result provides useful alternative descriptions of $\mathrm{T}_{(e, x)} \mathrm{S}$.

4.2. Proposition. Assume that $(e, x) \in \mathrm{S}$, and let $v \in Z^{-}(e)$ and $y \in Z$. Then the following conditions are equivalent: i) $(v, y) \in T_{(e, x)} \mathrm{S}$, ii) $P_{0}(e) y=0$ and $P_{1 / 2}(e) y=2\left\{P_{1 / 2}(e) v, e, x\right\}$, iii) $y=\{v e x\}+\{e v x\}+\{e e y\}$.

Proof. “i) $\Leftrightarrow$ ii)” Since $F^{-}(e) h=h$ for all $h \in Z^{-}(e)$, an elementary calculation gives

$$
\begin{gathered}
T_{(e, x)} \mathrm{S}=\Phi_{(e, x)}^{\prime}(00)\left(Z^{-}(e) \times Z_{1}(e)\right)= \\
\left\{(v, y) \in Z \times Z: v \in Z^{-}(e), y-K(e, v) x \in Z_{1}(e)\right\}
\end{gathered}
$$

From the definition of $K(e, v)$ we get for $v=i a+h \in Z^{-}(e)=i A(e) \oplus Z_{1 / 2}(e)$

$$
K(e, v) x=K(e, h) x+K(e, i a) x \in 2\{e, h, x\}+Z_{1}(e)
$$

hence if $y=y_{1}+y_{1 / 2}+y_{0}$ is the e-Peirce decomposition of $y$,

$$
\begin{aligned}
& y-K(e, v) x \in Z_{1}(e) \Longleftrightarrow y-2\{h, e, x\} \in Z_{1}(e) \\
& \Longleftrightarrow\left(P_{0}(e) y=0 \quad \text { and } \quad y_{1 / 2}=2\left\{P_{1 / 2}(e) v, e, x\right\}\right) .
\end{aligned}
$$

'i) $\Leftrightarrow$ iii)" As above, this is straightforward by Peirce arithmetics if we consider the various components in the Peirce subspaces of the equations on $y$.

4.3. Corollary. Given a pair $(e, x) \in \mathrm{S}$, the set $\left\{\left(P_{1 / 2}(e) D(a, b) e, D(a, b) x\right):(a, b) \in Z \times Z\right\}$ is contained in the tangent space $\mathrm{T}_{(e, x)} \mathrm{S}$.

Proof. Let $a, b \in Z$ and consider the pair $(v, y)$ where $v:=P_{1 / 2}(e) D(a, b) e$ and $y:=D(a, b) x$. It suffices to verify that $y=\{v e x\}+\{e v x\}+\{e e y\}$. Since the Peirce subspaces of $e$ span $Z$, we may even restrict ourselves to the cases when $a \in Z_{\lambda}(e), b \in Z_{\mu}(e),(\lambda, \mu \in\{0,1 / 2,1\})$. This is straightforward again by the Jordan identity.

4.4. Proposition. Let $V: \mathrm{M} \rightarrow \mathrm{TM}$ and $Y: Z \rightarrow \mathrm{T} Z$ be smooth vector fields on $\mathrm{M}$ and $Z$. Assume that $V$ is tangent to $\mathrm{M}$. Then the following statements are equivalent

i) For all $(e, x) \in \mathrm{S}$ we have $(\exp t Y) x \in Z_{1}((\exp t V) e),\left(t \in I_{e, x}\right)$, with some interval $I_{e, x}$ around 0. 
ii) $Y(x)=\{V(e), e, x\}+\{e, V(e), x\}+\{e, e, Y(x)\}$ whenever $(e, x) \in \mathrm{S}$.

If the vector fields $V$ and $Y$ are complete in $\mathrm{M}$ and $Z$ respectively, that is if $I_{e, x}=\mathbb{R}$ for all $(e, x) \in \mathrm{M} \times Z$, then i) and ii) are equivalent to

$$
\left.(\exp t Y) Z_{1}(e)=Z_{1}((\exp t V) e) \quad(e, x) \in \mathrm{S} t \in \mathbb{R}\right) .
$$

Proof. In terms of the manifold S, statement i) means that the vector field

$$
W(e, x):=(V(e), Y(x)) \quad(e, x) \in \mathrm{S}
$$

is tangent to $\mathrm{S}$. By Proposition 4.2, $W$ is a tangent vector field to $\mathrm{S}$ if and only if ii) holds.

Assume $V$ and $Y$ are complete. Then iii) makes sense and the implication iii) " $\Rightarrow$ "i) is trivial. Assume i) holds. Then $(\exp t Y) Z_{1}(e) \subset Z_{1}((\exp t V) e)$ for all $t \in \mathbb{R}$ and $e \in \mathrm{M}$. We can apply this argument with $(\exp t V) e$ in place of $e,(\exp t Y) x$ in place of $x$ and $-t$ in place of $t$. Hence we get

$$
(\exp (-t Y)) Z_{1}((\exp t V) e) \subset Z_{1}((\exp (-t V))(\exp t V) e)=Z_{1}(e)
$$

That is, we have also $Z_{1}((\exp t V) e) \subset(\exp t Y) Z_{1}(e)$.

\section{Equivariant vector fields on $M$}

Now we consider smooth vector fields on $\mathrm{M}$ and their relationship with those on the manifold of tripotents $M$. Fix a point $\mathbf{e} \in \mathbb{M}$ and recall that the tangent space $\mathrm{T}_{e} \mathbb{M}$ to $\mathbb{M}$ at $\mathbf{e}$ is

$$
\mathrm{T}_{e} \mathbb{M}=\mathfrak{D}_{1}(e)=\left\{K(e, u): u \in Z_{1 / 2}(e)\right\} \approx Z_{1 / 2}(e) .
$$

Each smooth vector field on $\mathbb{M}$ can be locally represented in a neighbourhood $\mathrm{N}$ of e either as a derivation-valued function $\mathfrak{X}: \mathrm{N} \rightarrow \mathfrak{D}_{1}(e)$ or as a vector-valued function $X: \mathrm{N} \rightarrow Z$, depending on whether we use $\mathfrak{D}_{1}(e)$ or $Z_{1 / 2}(e)$ as local coordinates for $\mathbb{M}$ at $\mathbf{e}$, and these two functions are related by

$$
\mathfrak{X}(\mathbf{e})=2(D(X(e), e)-D(e, X(e)) \quad X(e)=(\mathfrak{X}(\mathbf{e})) e
$$

due to the isomorphism $\mathfrak{D}_{1}(e) \leftrightarrow Z_{1 / 2}(e)$ constructed in (3.1). Remark that here $X: \mathrm{N} \rightarrow Z$ takes values in $Z_{1 / 2}(e)$, a direct summand in $\mathrm{T}_{e} \mathrm{M}$. Remark also that different smooth functions $X, Y: \mathrm{N} \rightarrow Z_{1 / 2}(e)$ may give rise to the same $\mathfrak{X}: \mathrm{N} \rightarrow \mathfrak{D}_{1}(e)$ via (9). Finally, note that for a vector field $X: \mathrm{N} \rightarrow Z$ with $X(e) \in Z_{1 / 2}(e)$ for all $e \in \mathrm{M}$, the following conditions are equivalent:

i) $K(e, X(e))=K(f, X(f))$ whenever $e \sim f \in \mathrm{M}$.

ii) $D(X(e), e)-D(e, X(e))=D(X(f), f)-D(f, X(f))$ whenever $e \sim f \in \mathrm{M}$.

iii) $D(X(e), e)+D(e, X(e))=D(X(f), f)+D(f, X(f))$ whenever $e \sim f \in \mathrm{M}$.

The equivalence i) $\Leftrightarrow$ ii) is obvious and ii) $\Leftrightarrow$ iii) follows from $e \sim f \Leftrightarrow(i e) \sim($ if $)$. These facts motivate the following discussion 
5.1. Definition. We say that a tangent vector field $X: \mathrm{M} \rightarrow \mathrm{TM}$ is equivariant if

$$
D(X(e), e)+D(e, X(e))=D(X(f), f)+D(f, X(f)) \quad(e \sim f \in \mathrm{M}) .
$$

The equivariant vector fields $X$ and $Y$ are said to be equivalent ( $X \approx Y$ in notation) if

$$
D(X(e), e)+D(e, X(e))=D(Y(e), e)+D(e, Y(e)) \quad(e \in \mathrm{M}) .
$$

Real-linear combinations of equivariant vector fields are equivariant. The pointwise limit of a sequence of equivariant vector fields is also equivariant.

5.2. Lemma. Let $X, Y: \mathrm{M} \rightarrow \mathrm{TM}$ be smooth vector fields on $\mathrm{M}$. Then

i) $X$ is equivariant if and only if $(\exp t X) e \sim(\exp t X) f$ whenever $e \sim f \in \mathrm{M}$ and $|t|<\varepsilon$ for some $\varepsilon>0$.

ii) $X \approx Y$ if and only if $(\exp t X) e \sim(\exp t Y) f$ whenever $e \sim f \in \mathrm{M}$ and $|t|<\varepsilon$ for some $\varepsilon>0$.

iii) $X \approx 0$ if and only if $X(e) \in i A(e),(e \in \mathrm{M})$.

Proof. Since $\mathrm{M}$ is a direct submanifold of $Z$, given any tripotent $e \in \mathrm{M}$, we have $(\exp t X) e \in$ $\mathrm{M}$ for sufficiently small real values of $t$. If $e \sim f \in \mathrm{M}$ and $(\exp t X) e \sim(\exp t Y) f$ for $t$ in a neighbourhood of 0 in $\mathbb{R}$ then the differentiation $\left.\frac{d}{d t}\right|_{t=0}$ of the relation $D((\exp t X) e)=$ $D((\exp t Y) f)$ yields $D(X(e), e)+D(e, X(e))=D(Y(f), f)+D(f, Y(f))$. This proves the implications " $\Leftarrow "$ in i) and ii). Let $X$ be equivariant, $e \sim f \in \mathrm{M}$ and let $(\exp t X) e$ and $(\exp t X) f$ be well-defined for $|t|<\varepsilon$. Consider the operators $\alpha_{t}:=i D((\exp t X) e) \in \mathbb{D}$ and $\beta_{t}:=i D((\exp t X) f) \in \mathbb{D},|t|<\varepsilon$. They the are solutions of the same initial value problem

$$
\frac{d}{d t} \gamma_{t}=W\left(\gamma_{t}\right) \quad \gamma_{0}=i D(e)=i D(f)
$$

where by the equivariance of $X, W(i D(g)):=i D(X(g), g)+i D(g, X(g)),(g \in \mathrm{M})$, is a well-defined tangent vector field to $\mathbb{D}$. Since $\mathbb{D}$ is a direct submanifold of $\operatorname{Der}(Z)$, the solution is unique and $\alpha_{t}=\beta_{t}$ and hence $(\exp t X) e \sim(\exp t X) f,|t|<\varepsilon$. This proves " $\Rightarrow$ " in i). The proof of " $\Rightarrow$ " in ii) is similar.

The implication " $\Leftarrow "$ in iii) is clear. If $X(e)=u+i a$ where $u \in Z_{1 / 2}(e)$ and $a \in A(e)$ then evaluating at $e$ the relation $D(X(e), e)+D(e, X(e))=0$ gives $u=0$.

5.3. Corollary. If $X$ is an equivariant complete smooth vector field on $\mathrm{M}$ then $\exp X$ maps equivalence classes of tripotents onto equivalence classes: $(\exp X) \mathbf{e} \in \mathbb{M}$ for any $\mathbf{e} \in \mathbb{M}$.

Recall that the complete smooth vector fields on $\mathrm{M}$ form a real Lie algebra with the pointwise vector space operations and the Poisson bracket

$$
[X, Y]:=Y_{X}^{\prime}-X_{Y}^{\prime}, \quad Y_{X}^{\prime}(e):=\left.\frac{d}{d t}\right|_{t=0} Y((\exp t X) e) \quad(e \in \mathrm{M}) .
$$

For a vector field $X: \mathrm{M} \rightarrow Z$ and a Peirce projector $P: Z \rightarrow Z$ we define $P X: \mathrm{M} \rightarrow Z$ by $P X(e):=P(e) X(e),(e \in \mathrm{M})$. As a consequence of the following result, the algebraic connection studied in [3] and [7],

$$
\left(\nabla_{X} Y\right)(e):=P_{1 / 2}(e) Y_{X}^{\prime}(e) \quad(e \in \mathrm{M}),
$$

preserves smooth equivariant complete vector fields of $\mathrm{M}$. 
5.4. Proposition. Let $X, Y: \mathrm{M} \rightarrow \mathrm{TM}$ be equivariant smooth vector fields. Then $P_{1 / 2} X \approx X$, and both $P_{1 / 2} X$ and $[X, Y]$ are equivariant. If $X=P_{1 / 2} X$ and $Y=P_{1 / 2} Y$ then also $\nabla_{X} Y$ is equivariant.

Proof. By 5.2 iii), $P_{1}^{-} X$ is equivalent to 0 . Since real linear combinations of equivariant vector fields are equivariant, $P_{1 / 2} X=X-P_{1}^{-} X$ is equivariant and $P_{1 / 2} X-X \approx 0$.

Let $e \sim f \in \mathrm{M}$ and set $e_{t}:=(\exp t X) e$ and $f_{t}:=(\exp t X) f$. We know that $e_{t} \sim f_{t}$, $(t \in \mathbb{R})$. Hence, for all $t \in \mathbb{R}$,

$$
D\left(Y\left(e_{t}\right), e_{t}\right)+D\left(e_{t}, Y\left(e_{t}\right)\right)=D\left(Y\left(f_{t}\right), f_{t}\right)+D\left(f_{t}, Y\left(f_{t}\right)\right)
$$

To shorten some lengthy formulas we set

$$
\begin{gathered}
\Delta_{X, Y}(e):=\left.\frac{d}{d t}\right|_{t=0}\left(D\left(Y\left(e_{t}\right), e_{t}\right)+D\left(e_{t}, Y\left(e_{t}\right)\right)\right)= \\
=\left(D\left(Y_{X}^{\prime}(e), e\right)+D\left(e, Y_{X}^{\prime}(e)\right)\right)+(D(X(e), Y(e))+D(Y(e), X(e))) .
\end{gathered}
$$

Note that $\Delta_{X, Y}(e)$ is a real-bilinear function of $X, Y$ and that the second summand in the above expression is symmetric in $X, Y$. By differentiating (10) at $t=0$, we get

$$
\Delta_{X, Y}(e)=\Delta_{X, Y}(f) \quad(e \sim f) .
$$

By subtracting the same equation where the roles of $X$ and $Y$ are interchanged, we conclude the equivariance of $[X, Y]$.

For $e \sim f$ we have $D(e)=D(f)$, hence the Lie adjoints satisfy $D(e)_{\#}=D(f)_{\#}$. Since both the projections $\Pi_{m}(e): \operatorname{Der}(Z) \rightarrow \operatorname{Der}(Z)$ introduced in (2) and

$$
\Pi(e):=\mathrm{Id}-\sum_{k=0}^{k=2} \Pi_{k}(e)
$$

are polynomials of $D(e)_{\#}^{2}$, we get $\Pi(e)=\Pi(f)$ for $e \sim f \in \mathrm{M}$. Let us write $Y_{X}^{\prime}(e)=$ $\sum_{k=0}^{k=2} V_{k}(e)$ and $z=\sum_{k=0}^{2} z_{k}$ for the $e$-Peirce decompositions of $Y_{X}^{\prime}(e)$ and $z \in Z$, and set

$$
L_{k}(e):=D\left(V_{k}(e), e\right)+D\left(e, V_{k}(e)\right) \quad(k=0,1,2) .
$$

By Peirce arithmetic

$$
P_{\lambda}(e) L_{1}(e) P_{\mu}(e) z=P_{\lambda}(e)\left(\left\{V_{1}(e), e, z_{\mu}\right\}+\left\{e, V_{1}(e), z_{\mu}\right\}\right) \in P_{\lambda}(e) Z_{\mu}(e)
$$

which shows that $P_{\lambda}(e) L_{1}(e) P_{\mu}(e)=0$ for $\lambda \neq \mu$ and $P_{\lambda}(e) L_{1}(e) P_{\lambda}(e)=L_{1}(e) P_{\lambda}(e)$. Thus

$$
\Pi(e) L_{1}(e)=L_{1}(e)-\sum_{k=0}^{k=2} P_{k}(e) L_{1}(e) P_{k}(e)=L_{1}(e)-\sum_{k=0}^{k=2} L_{1}(e) P_{k}(e)=0 .
$$

In a similar manner we obtain $\Pi(e) L_{1 / 2}(e)=L_{1 / 2}(e)$ and $\Pi(e) L_{0}(e)=0$, hence

$$
\Pi(e)\left[D\left(Y_{X}^{\prime}(e), e\right)+D\left(e, Y_{X}^{\prime}(e)\right)\right]=D\left(P_{1 / 2}(e) Y_{X}^{\prime}(e), e\right)+D\left(e, P_{1 / 2}(e) Y_{X}^{\prime}(e)\right) .
$$


From the assumptions $X=P_{1 / 2} X$ and $Y=P_{1 / 2} Y$ we get by Peirce arithmetic as before

$$
\Pi(e) D(X(e), Y(e))=\Pi(e) D(Y(e), X(e))=0
$$

and so

$$
\Pi(e) \Delta_{X, Y}(e)=D\left(P_{1 / 2}(e) Y_{X}^{\prime}(e), e\right)+D\left(e, P_{1 / 2}(e) Y_{X}^{\prime}(e)\right) .
$$

The same equation holds with $f$ in place of $e$. Since $\Pi(e)=\Pi(f)$ an application of $\Pi(e)$ to both sides of (11) yields that $e \mapsto P_{1 / 2}(e) Y_{X}^{\prime}(e)$ is equivariant.

5.5. Theorem. Let $X, Y$ and $\widetilde{X}, \widetilde{Y}$ be smooth equivariant vector fields on $\mathrm{M}$. If $X \approx \widetilde{X}$ and $Y \approx \widetilde{Y}$ then $[X, Y] \approx[\widetilde{X}, \widetilde{Y}]$. The family $\mathfrak{E}(\mathrm{M})$ of all smooth equivariant vector fields $X$ on $\mathrm{M}$ such that $P_{1 / 2} X=X$ forms a Lie algebra with the product

$$
[X, Y]_{*}:=\nabla_{X} Y-\nabla_{Y} X=P_{1 / 2}[X, Y] .
$$

Proof. It is well-known [16] that

$$
[X, Y] e=\left.\frac{1}{2} \frac{d^{2}}{d t^{2}}\right|_{t=0}(\exp t Y)(\exp (-t X))(\exp (-t Y))(\exp t X) e \quad(e \in \mathrm{M})
$$

Let $X \approx \tilde{X}, Y \approx \widetilde{Y}$ and assume that $t$ is sufficiently small. By 5.2 iii)), the values of the exponential expression on right hand side of the above formula corresponding to the pairs $(X, Y)$ and $(\widetilde{X}, \widetilde{Y})$ lie on the same class of equivalence. Hence $[X, Y] \approx[\widetilde{X}, \widetilde{Y}]$. The Jacobi identity for $[\cdot, \cdot]_{*}$ follows from this. Let $C, X, Y \in \mathfrak{E}(\mathrm{M})$. Then

$$
\left[C,[X, Y]_{*}\right]_{*}=P_{1 / 2}\left[C,[X, Y]_{*}\right] \approx\left[C,[X, Y]_{*}\right]=\left[C, P_{1 / 2}[X, Y]\right] \approx[C,[X, Y]] .
$$

Therefore $\left[C,[X, Y]_{*}\right]_{*}=P_{1 / 2}[C,[X, Y]]$. Similarly $\left[[C, X]_{*}, Y\right]_{*}=P_{1 / 2}[[C, X], Y]$ and $\left[X,[C, Y]_{*}\right]_{*}=P_{1 / 2}[X,[C, Y]]$. Thus we can deduce the Jacobi identity $\left[C,[X, Y]_{*}\right]_{*}=$ $\left[[C, X]_{*}, Y\right]_{*}+\left[X,[C, Y]_{*}\right]_{*}$ from that of $[.,$.$] by multiplying the latter by P_{1 / 2}$.

5.6. Corollary. The Lie algebra $\mathfrak{D}(\mathbb{M})$ of smooth vector fields on $\mathbb{M}$ is isomorphic to $\mathfrak{E}(\mathrm{M})$, the Lie algebra of the smooth equivariant vector fields $X$ on $\mathrm{M}$ that satisfy $P_{1 / 2} X=X$, by the map

$$
\mathfrak{X} \mapsto X \quad \text { where } \quad X:=[e \mapsto(\mathfrak{X}(\mathbf{e})) e] \quad \mathfrak{X} \in \mathfrak{D}(\mathbb{M})
$$

whose inverse is

$$
X \mapsto \mathfrak{X} \quad \text { where } \quad \mathfrak{X}(\mathbf{e})=2(D(X(e), e)-D(e, X(e)) \quad X \in \mathfrak{D}(\mathrm{M}) .
$$

5.7. Corollary. Given a smooth complete tangent vector field $V: \mathrm{M} \rightarrow \mathrm{TM}$ to $\mathrm{M}$, if there exists a smooth complete vector field $Y: Z \rightarrow \mathrm{T} Z$ such that 4.4 ii) holds then $V$ is equivariant.

Proof. Suppose 4.4 ii) holds. Then we have 4.4 iii) as well. Consider two equivalent tripotents $e \sim f \in$ M. Since $Z_{1}(e)=Z_{1}(f)$, it follows

$$
Z_{1}((\exp t V) e)=(\exp t Y) Z_{1}(e)=(\exp t Y) Z_{1}(f)=Z_{1}((\exp t V) f) .
$$

Thus $(\exp t V) e \sim(\exp t V) f$, that is $Y((\exp t V) e)=Y((\exp t V) f),(t \in \mathbb{R})$. By taking the derivative $\left.\frac{d}{d t}\right|_{t=0}$, we get $D(V(e), e)+D(e, V(e))=D(V(f), f)+D(f, V(f))$. 


\section{Complex manifold structure on $\mathrm{IM}$}

In this section we describe the holomorphic manifold structures of $\mathbb{M}$ and $\mathbb{D}$ and prove that they are isomorphic to $\mathbb{P}$. In particular we prove that given a continuous vector field $V$ on $\mathbb{P}$ there exists a unique continuous mapping $V^{*}: \mathrm{M} \rightarrow Z$ with values in $Z_{1 / 2}(e)$ such that

$$
V^{*}(e) \in Z_{1 / 2}(e) \quad \text { and } \quad(\exp t V) J_{e}=J_{\left(\exp t V^{*}\right) e} \quad(e \in \mathrm{M}, t \in \mathbb{R}) .
$$

6.1. Theorem. Let $Z$ and $\mathrm{M}$ be a JB*-triple and the manifold of its non zero tripotents. Then for each $e \in \mathrm{M}$ there exists a neighborhood $W$ of 0 in $Z_{1 / 2}(e)$ and an invertible real-analytic map $Y_{e}: W \rightarrow \mathrm{M}$ such that

$$
Y_{e}(0)=e, \quad(\exp D(u, e)) J_{e}=J_{Y_{e}(u)} \quad(u \in W)
$$

Proof. Fix any $e \in \mathrm{M}$ and any $u \in Z_{1 / 2}(e)$. The vector field $C_{u}^{(e)}: e^{\prime} \mapsto P_{1 / 2}\left(e^{\prime}\right) D(u, e) e^{\prime}$, $\left(e^{\prime} \in \mathrm{M}\right)$, is tangent to $\mathrm{M}$ which is a direct submanifold of $Z$, hence the exponential of $C_{u}^{(e)}$ is a well-defined mapping $\mathrm{M} \rightarrow \mathrm{M}$. Define

$$
Y_{e}(u):=\left(\exp C_{u}^{(e)}\right) e \quad\left(u \in Z_{1 / 2}(e)\right)
$$

Then the curve $t \mapsto e_{t}:=Y_{e}(t u),(t \in \mathbb{R})$, is the solution of the initial value problem $e_{0}=e$, $\frac{d}{d t} e_{t}=P_{1 / 2}\left(e_{t}\right) D(u, e) e_{t}$. Define $F: \mathrm{S} \rightarrow Z \times Z$ on the manifold $\mathrm{S}$ of Theorem 4.1 as follows

$$
F\left(e^{\prime}, x^{\prime}\right):=\left(P_{1 / 2}\left(e^{\prime}\right) D(u, e) e^{\prime}, D(u, e) x^{\prime}\right) \quad\left(e^{\prime}, x^{\prime}\right) \in \mathrm{S} .
$$

Consider any $\left(e^{\prime}, x^{\prime}\right) \in \mathrm{S}$. We can apply Corollary 4.3 with $(e, x)$ replaced with $\left(e^{\prime}, x^{\prime}\right)$ and $(a, b)$ replaced with $(u, e)$. Hence we conclude that

$$
\left(P_{1 / 2}\left(e^{\prime}\right) D(u, e) e^{\prime}, \quad D(u, e) x^{\prime}\right) \in \mathrm{T}_{(e, x)} \mathrm{S} .
$$

This means that $F$ is a tangent vector field to $\mathrm{S}$ and its exponential is a well-defined mapping $\mathrm{S} \rightarrow \mathrm{S}$. In particular, there is a curve $t \mapsto\left(e_{t}^{\prime}, x_{t}^{\prime}\right) \in \mathrm{S},(t \in \mathbb{R})$, such that $\left(e_{0}^{\prime}, x_{0}^{\prime}\right)=(e, e)$ and $\frac{d}{d t}\left(e_{t}^{\prime}, x_{t}^{\prime}\right)=F\left(e_{t}^{\prime}, x_{t}^{\prime}\right)$. Then

$$
\left\{\begin{array} { l } 
{ \frac { d } { d t } e _ { t } ^ { \prime } = P _ { 1 / 2 } ( e _ { t } ^ { \prime } ) D ( u , e ) e _ { t } ^ { \prime } } \\
{ e _ { 0 } ^ { \prime } = e }
\end{array} \quad \left\{\begin{array}{l}
\frac{d}{d t} x_{t}^{\prime}=D(u, e) x_{t}^{\prime} \\
x_{0}^{\prime}=e
\end{array}\right.\right.
$$

By the uniqueness of solutions of initial value problems, $e_{t}^{\prime}=e_{t}$ and $x_{t}^{\prime}=(\exp t D(u, e)) e$ for all $t \in \mathbb{R}$. Since $\left(e_{t}^{\prime}, x_{t}^{\prime}\right) \in \mathrm{S}$, we have $x_{t}^{\prime} \in Z_{1}\left(e_{t}^{\prime}\right)$ for all $t \in \mathbb{R}$. In particular, for $t=1$

$$
x_{1}^{\prime} \in Z_{1}\left(e_{1}^{\prime}\right)=Z_{1}\left(Y_{e}(u)\right) \text {. }
$$

Note that $x_{1}^{\prime}$ is a von Neumann regular element, hence the inner ideal generated by $x_{1}^{\prime}$, that is $J_{x_{1}^{\prime}}$, is contained in $Z_{1}\left(Y_{e}(u)\right)=J_{Y_{e}(u)}$,

$$
J_{(\exp D(u, e)) e} \subset J_{Y_{e}(u)}
$$


Since clearly $Y_{e}$ is invertible and $Y_{e}^{-1}(u)=Y_{e}(-u)$, the same argument with $Y_{e}^{-1}$ gives that above we have equality,

$$
J_{(\exp D(u, e)) e}=J_{Y_{e}(u)}
$$

By definition of the local chart of $\mathbb{P}$ at $J_{e}$ we have $J_{(\exp D(u, e)) e}=\exp D(u, e) J_{e}$ which replaced in the latter gives (13). This completes the proof.

Remark that by definition we have $Y_{e}(u)=\left(\exp C_{u}^{(e)}\right) e$, hence

$$
\exp D(u, e) J_{e}=J_{\left(\exp C_{u}^{(e)}\right) e} \quad(u \in W)
$$

The smooth vector fields $C_{u}^{(e)}: e^{\prime} \mapsto P_{1 / 2}\left(e^{\prime}\right) D(u, e) e^{\prime},\left(e^{\prime} \in \mathrm{M}\right)$, and $Y_{u}^{(e)}: z \mapsto D(u, e) z$, $(z \in Z)$, considered in the above proof are tangent to $\mathrm{M}$ and $Z$, and they are bounded on $\mathrm{M}$ and $Z$ respectively. As a consequence of the boundedness, they are complete on $\mathrm{M}$ and $Z$, and the pair $\left(C_{u}^{(e)}, Y_{u}^{(e)}\right)$ satisfies the assumptions in Corollary (5.7), hence $C_{u}^{(e)}$ is equivariant on $\mathrm{M}$, which together with (14) solves problem (12).

6.2. Remark. In terms of $\mathbb{P}$ this result can be interpreted as follows. Let $e_{0} \in \mathrm{M}$ be any given tripotent (used as base point for M). For each vector $u \in Z_{1 / 2}\left(e_{0}\right)$,

$$
C_{u}^{\left(e_{0}\right)}: e \mapsto P_{1 / 2}(e) D\left(u, e_{0}\right) e \quad(e \in \mathrm{M})
$$

is a bounded smooth equivariant complete tangent vector field to M. Then the map

$$
\widetilde{Y}^{\left(e_{0}\right)}(u):=\pi\left[\left(\exp C_{u}^{\left(e_{0}\right)}\right) e_{0}\right]=\pi Y_{e_{0}}(u) \quad\left(u \in Z_{1 / 2}\left(e_{0}\right)\right)
$$

is a local chart of $\mathbb{M}$ around the equivalence class $\pi\left(e_{0}\right)$. The map

$$
\bar{Y}^{\left(e_{0}\right)}(u):=Z_{1}\left(\left(\exp C_{u}^{\left(e_{0}\right)}\right) e_{0}\right) \quad\left(u \in Z_{1 / 2}\left(e_{0}\right)\right)
$$

is a local chart of $\mathbb{P}$ around the principal ideal $J_{e_{0}}=Z_{1}\left(e_{0}\right)$. The map

$$
\widehat{Y}^{\left(e_{0}\right)}(u):=i D\left(\left(\exp C_{u}^{\left(e_{0}\right)}\right) e_{0}\right) \quad\left(u \in Z_{1 / 2}\left(e_{0}\right)\right)
$$

is a local chart of $\mathbb{D}$ around the derivation $i D\left(e_{0}\right)$. Thus we have the commutative diagram

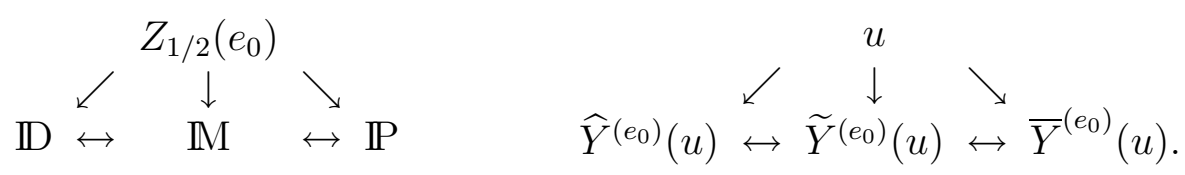

6.3. Corollary. The families of maps $\widetilde{\mathcal{Y}}:=\left\{\widetilde{Y}^{\left(e_{0}\right)}: e_{0} \in \mathrm{M}\right\}$ and $\widehat{\mathcal{Y}}:=\left\{\widehat{Y}^{\left(e_{0}\right)}: e_{0} \in \mathrm{M}\right\}$, where $\widetilde{Y}^{\left(e_{0}\right)}$ and $\widehat{Y}^{\left(e_{0}\right)}$ are given by (15) and (17) respectively, are holomorphic atlases for the manifolds $\mathbb{M}$ and $\mathbb{D}$ both of which are isomorphic to $\mathbb{P}$ via the the bijections $\mathbf{e} \leftrightarrow J_{e} \leftrightarrow i D(e)$. 
Proof. By Kaup's construction [9], the family $\overline{\mathcal{Y}}:=\left\{\bar{Y}^{\left(e_{0}\right)}: e_{0} \in \mathrm{M}\right\}$ is a holomorphic atlas for $\mathbb{P}$, hence the transition maps $\left[\bar{Y}^{\left(e_{0}\right)}\right]^{-1} \circ \bar{Y}^{\left(f_{0}\right)},\left(e_{0}, f_{0} \in \mathrm{M}\right)$, are holomorphic. Since the above diagrams are commutative, also the maps $\left[\widetilde{Y}^{\left(e_{0}\right)}\right]^{-1} \circ \widetilde{Y}^{\left(f_{0}\right)}$ and $\left[\widehat{Y}^{\left(e_{0}\right)}\right]^{-1} \circ \widehat{Y}^{\left(f_{0}\right)}$ are holomorphic. Thus $\mathrm{M}$ and $\mathrm{D}$ are holomorphic manifolds in the above atlases and it is easy to show that the bijections $\mathbf{e} \leftrightarrow J_{e} \leftrightarrow i D(e)$ are isomorphisms and that $\pi: \mathrm{M} \rightarrow \mathbb{M}$ is a real-analytic open submersion. In particular, by Godement's theorem ([16] Theorem 8.14) $\mathbb{M}$ hence $\mathbb{P}$ carries the quotient manifold structure of M relative to $\sim$.

Recall that given any element $x \in \operatorname{Reg}(Z)$ and the principal inner ideal $J_{x}$ generated by $x$ in $Z$, we have $J_{x}=J_{s(x)}$ where $s(x)$ is a tripotent given by $s(x):=\lim _{n \rightarrow \infty} c^{n}(x)$ and $c(x)$ stands for the cubic root operation defined by defined by the odd functional calculus.

6.4. Theorem. Let $Z$ and $\mathrm{M}$ be a JC*-triple (that is, $Z$ admits only the trivial $\mathrm{JB}^{*}$-triple representation in the triple of $3 \times 3$ hermitian octonion matrices) and the manifold of its non zero tripotents. Let $e \in \mathrm{M}$ and let $Y_{e}: W \rightarrow \mathrm{M}$ be the analytic function constructed in theorem (6.1). Then $Y_{e}(u)$ is the support tripotent of $[\exp t D(e, u)]$ e for all $u \in W \subset Z_{1 / 2}(e)$.

Proof. Let $e \in \mathrm{M}$ and $u \in Z_{1 / 2}(e)$ be arbitrarily given. By writing $x_{t}:=[\exp t D(u, e)] e$ and $s_{t}$ for the support tripotent of $x_{t}$ we have to verify that

$$
\frac{d}{d t} s_{t}=P_{1 / 2}\left(s_{t}\right)\left\{u e s_{t}\right\} \quad(t \in \mathbb{R}) .
$$

Since $Z$ is a $\mathrm{JC}^{*}$-triple, it is well-known we may assume without loss of generality that $Z=$ $\mathcal{L}(H)$ where $H$ is a Hilbert space, $H=H_{1} \oplus^{2} H_{2}$ with some subspaces $H_{1}, H_{2}$ and

$$
e=\left(\begin{array}{cc}
I & 0 \\
0 & 0
\end{array}\right), \quad u=2\left(\begin{array}{cc}
0 & A \\
B & 0
\end{array}\right)
$$

where $I=$ [identity on $\left.H_{1}\right], A \in \mathcal{L}\left(H_{2}, H_{1}\right)$ and $B \in \mathcal{L}\left(H_{1}, H_{2}\right)$. Then we have

$$
x_{t}:=\sum_{k=0}^{2} \frac{t^{k}}{k !} D(u, e)^{k} e=\left(\begin{array}{cc}
I & t A \\
t B & t^{2} B A
\end{array}\right)=\ell_{t} r_{t}
$$

with the operator matrices $\ell_{t}:=\left(\begin{array}{c}I \\ t B\end{array}\right)$ and $r_{t}:=\left(\begin{array}{ll}I t A\end{array}\right)$. For an operator $a \in \mathcal{L}(H)$, we let $a=u|a|$ denote the polar decomposition of $a$ in $\mathcal{L}(H)$. If $a$ is von Neumann regular in $\mathcal{L}(H)$ then it has a Moore-Penrose inverse $a^{\dagger}$ and the tripotent support $s(a)$ of $a$ is the partial isometry $u$ which is given by $u=a\left(a^{*} a\right)^{\dagger}$. In our case both $l_{t}$ and $r_{t}$ have Moore-Penrose inverses

$$
\ell_{t}^{\dagger}=\left[I+t^{2} B^{*} B\right]^{-1}\left(\begin{array}{ll}
I & \left.t B^{*}\right)
\end{array} r_{t}^{\dagger}=\left(\begin{array}{c}
I \\
t A^{*}
\end{array}\right)\left[I+t^{2} A A^{*}\right]^{-1} .\right.
$$

An elementary calculation with generalized inverses gives (see [2])

$$
s_{t}=\ell_{t}\left(\ell_{t}^{*} \ell_{t}\right)^{-1 / 2}\left(r_{t} r_{t}^{*}\right)^{-1 / 2} r_{t} \quad(t \in \mathbb{R}) .
$$

Let us calculate $\frac{d}{d t} s_{t}$ and $P_{1 / 2}\left(s_{t}\right)\left\{u e s_{t}\right\}$ in terms of $\ell_{t}$ and $r_{t}$. We have

$$
\left\{u e s_{t}\right\}=\frac{1}{2} u e s_{t}+\frac{1}{2} s_{t} e u=\left(\begin{array}{cc}
0 & 0 \\
B & 0
\end{array}\right) s_{t}+s_{t}\left(\begin{array}{cc}
0 & A \\
0 & 0
\end{array}\right)
$$


hence in particular $\left\{u e s_{t}\right\} \in Z_{1 / 2}\left(s_{t}\right) \oplus Z_{1}\left(s_{t}\right)$, and therefore

$$
P_{1 / 2}\left(s_{t}\right)\left\{u e s_{t}\right\}=\left\{u e s_{t}\right\}-P_{1}\left(s_{t}\right)\left\{u e s_{t}\right\}=\left\{u e s_{t}\right\}-s_{t} s_{t}^{*}\left\{u e s_{t}\right\} s_{t}^{*} s_{t} .
$$

Thus, since $s_{t} s_{t}^{*} s_{t}=\left\{s_{t} s_{t} s_{t}\right\}=s_{t}$,

$$
\begin{gathered}
P_{1 / 2}\left(s_{t}\right)\left\{\text { ues } s_{t}\right\} \\
\left(\begin{array}{cc}
0 & 0 \\
B & 0
\end{array}\right) s_{t}-s_{t} s_{t}^{*}\left(\begin{array}{cc}
0 & 0 \\
B & 0
\end{array}\right) s_{t}+s_{t}\left(\begin{array}{cc}
0 & A \\
0 & 0
\end{array}\right)-s_{t}\left(\begin{array}{cc}
0 & A \\
0 & 0
\end{array}\right) s_{t}^{*} s_{t} .
\end{gathered}
$$

It is convenient to introduce the operator matrices

$$
d_{X}:=\operatorname{diag}\left(\left[I+t^{2} X^{*} X\right]^{-1 / 2},\left[I+t^{2} X X^{*}\right]^{-1 / 2}\right)=\left(\begin{array}{cc}
{\left[I+t^{2} X^{*} X\right]^{-1 / 2}} & 0 \\
0 & {\left[I+t^{2} X X^{*}\right]^{-1 / 2}}
\end{array}\right)
$$

with $X \in \mathcal{L}\left(H_{1}, H_{2}\right)$. In general, if we have a power series $\varphi(\xi)=\sum_{k=0}^{\infty} \alpha_{k} \xi^{k}$ with complex coefficients, by functional calculus $X \varphi\left(X^{*} X\right)=\varphi\left(X X^{*}\right) X,\left(X \in \mathcal{L}\left(H_{2}, H_{1}\right)\right)$. On the basis of this observation we can write

$$
\begin{aligned}
& \ell_{t}\left(\ell_{t}^{*} \ell_{t}\right)^{-1 / 2}=d_{t B^{*}} \ell_{t}, \quad\left(r_{t}^{*} r_{t}\right)^{-1 / 2} r_{t}=r_{t} d_{t A}, \quad s_{t}=d_{t B^{*}} \ell_{t} r_{t} d_{t A}, \\
& s_{t} s_{t}^{*}=\ell_{t}\left(\ell_{t}^{*} \ell_{t}\right)^{-1} \ell_{t}^{*}=d_{t B^{*}}^{2} \ell_{t} \ell_{t}^{*}, \quad s_{t}^{*} s_{t}=r_{t}^{*}\left(r_{t} r_{t}^{*}\right)^{-1} r_{t}=r_{t}^{*} r_{t} d_{t A}^{2} .
\end{aligned}
$$

It follows

$$
\begin{aligned}
\text { ues }_{t}= & \left(\begin{array}{cc}
0 & 0 \\
B & 0
\end{array}\right) d_{t B^{*}} \ell_{t} r_{t} d_{t A}=d_{t B^{*}}\left(\begin{array}{cc}
0 & 0 \\
B & 0
\end{array}\right) \ell_{t} r_{t} d_{t A}= \\
& d_{t B^{*}}\left(\begin{array}{c}
0 \\
B
\end{array}\right) r_{t} d_{t A}=d_{t B^{*}}\left[\frac{d}{d t} \ell_{t}\right] r_{t} d_{t A}
\end{aligned}
$$

Similarly $s_{t} e u=d_{t B^{*}} \ell_{t}\left[\frac{d}{d t} d_{t A}\right]$. Hence since $\ell_{t} \ell_{t}^{*} d_{t B^{*}}=d_{t B^{*}} \ell_{t} \ell_{t}^{*}$, we get also

$$
\begin{aligned}
& s_{t} s_{t}^{*} u e s_{t}=d_{t B^{*}}^{2} \ell_{t} \ell_{t}^{*} d_{t B^{*}}\left(\begin{array}{c}
0 \\
B
\end{array}\right) r_{t} d_{t A}=d_{t B^{*}}^{3} \ell_{t} \ell_{t}^{*}\left(\begin{array}{c}
0 \\
B
\end{array}\right) r_{t} d_{t A}= \\
& d_{t B^{*}}^{3}\left(t B^{*} B t^{2} B^{*} B B^{*}\right) r_{t} d_{t A}=d_{t B^{*}}^{3}\left(\begin{array}{cc}
t B^{*} B & t^{2} B^{*} B A \\
t^{2} B B^{*} B & t^{3} B B^{*} B A
\end{array}\right) d_{t A}= \\
= & \operatorname{diag}\left(\left[I+t^{2} B^{*} B\right]^{-3 / 2} t B^{*} B,\left[I+t^{2} B B^{*}\right]^{-3 / 2} t B B^{*}\right)\left(\begin{array}{cc}
I & t A \\
t B & t^{2} B A
\end{array}\right) d_{t A}= \\
= & {\left[-\frac{d}{d t} d_{t B^{*}}\right] \ell_{t} r_{t} d_{t A} . }
\end{aligned}
$$

Similarly $d_{t B^{*}} \ell_{t} r_{t}\left[-\frac{d}{d t} d_{t A}\right]=s_{t} e u s_{t}^{*} s_{t}$. Therefore we conclude

$$
\begin{gathered}
\frac{d}{d t} s_{t}=\frac{d}{d t}\left[d_{t B^{*}} \ell_{t} r_{t} d_{t A}\right]= \\
{\left[\frac{d}{d t} d_{t B^{*}}\right] \ell_{t} r_{t} d_{t A}+d_{t B^{*}}\left[\frac{d}{d t} \ell_{t}\right] r_{t} d_{t A}+d_{t B^{*}} \ell_{t}\left[\frac{d}{d t} r_{t} d_{t A}\right]+d_{t B^{*}} \ell_{t} r_{t}\left[\frac{d}{d t} d_{t A}\right]} \\
=-s_{t} s_{t}^{*} u e s_{t}+u e s_{t}+s_{t} e u-s_{t} e u s_{t}^{*} s_{t}=P_{1 / 2}\left(s_{t}\right)\left\{u e s_{t}\right\}
\end{gathered}
$$


Recall from [10] that for von Neumann regular elements $a \in \operatorname{Reg}(Z)$ it is possible to define the Peirce projectors $P_{k}(a)$, the Peirce subspaces $Z_{k}(a),(k \in\{0,1 / 2,1\})$, and the equivalence $\sim$ in the sense of Neher, all these notions being consistent with their analogs for tripotents. Recall also that $\mathrm{R}:=\operatorname{Reg}(Z) \backslash\{0\}$ and $\mathrm{M}:=\operatorname{Tri}(Z) \backslash\{0\}$ are complex (respectively, real) analytic direct submanifolds of $Z$ with tangent spaces $\mathrm{T}_{a} \mathrm{R}=Z_{1}(a) \oplus Z_{1 / 2}(a),(a \in \mathrm{R})$ and $\mathrm{T}_{e} \mathrm{M}=i A(e) \oplus Z_{1 / 2}(e),(e \in \mathrm{M})$. Restricting the corresponding local charts to the direct summands $Z_{1 / 2}(a)$ and $Z_{1 / 2}(e)$ we get a complex (respectively, a real) analytic submanifold $R_{1} \subset \mathrm{R}$ and $\mathrm{M}_{1} \subset \mathrm{M}$ with $\mathrm{M}_{1} \subset \mathrm{R}_{1}$. It is known that the support function $s: \mathrm{R} \rightarrow \mathrm{M}$ is not continuous. However, as a consequence of (6.1) and (6.4), if $Z$ is a JC${ }^{*}$-triple then the restriction $\left.s\right|_{R_{1}}: R_{1} \rightarrow M_{1} \subset R_{1}$ is a real analytic retraction of $R_{1}$ onto $M_{1}$.

\section{Holomorphic maps on $\mathbb{P}$}

In this section we study the relationship between holomorphy on $\mathbb{P}$ and holomorphy on $Z$. Recall from [10] that for von Neumann regular elements $a \in \operatorname{Reg}(Z)$ the tangent space to $\operatorname{Reg}(Z)$ at $a$ is $\mathrm{T}_{a} \mathrm{R}=Z_{1}(a) \oplus Z_{1 / 2}(a)$ and a local chart is

$$
\Psi:(u, v) \mapsto(\exp D(u+v, a)) a \quad(u, v) \in Z_{1}(a) \oplus Z_{1 / 2}(a) .
$$

In particular, there is a neighbourhood $N$ of $a \in \mathrm{R}$ such that for each $x \in N$ there exists a unique pair $(u(x), v(x)) \in Z_{1}(a) \times Z_{1 / 2}(a)$ for which $x=(\exp D(u+v, a)) a$, and the map $x \rightarrow v(x)$ is holomorphic. Finally, for $x, x^{\prime}$ in the neighbourhood $N$, we have $x \sim x^{\prime}$ if and only if $v(x)=v\left(x^{\prime}\right)$.

7.1. Lemma. Let $e \in \mathrm{M}$ and let $\Phi: \mathbb{M} \rightarrow \mathbb{C}$ be a mapping which is differentiable in the real sense at the point $\mathbf{e}$. Then $\Phi$ is $\mathbb{C}$-differentiable at $\mathbf{e}$ if and only if

$$
\left.\frac{d}{d t}\right|_{t=0} \Phi\left(\pi\left[\left(\exp t C_{i u}^{(e)}\right) e\right]\right)=\left.i \frac{d}{d t}\right|_{t=0} \Phi\left(\pi\left[\left(\exp t C_{u}^{(e)}\right) e\right]\right) \quad\left(u \in Z_{1 / 2}(e)\right) .
$$

Proof. By definition, $\Phi$ is $\mathbb{C}$-differentiable at e if and only if $\Phi \circ \tilde{Y}^{(e)}: Z_{1 / 2}(e) \rightarrow \mathbb{C}$ is $\mathbb{C}$ differentiable at 0 . The $\mathbb{R}$-differentiability of $\Phi$ at $\mathbf{e}=\widetilde{X}^{(e)}(0)$ means that the mapping $\Phi \circ$ $\widetilde{Y}^{(e)}: Z_{1 / 2}(e) \rightarrow \mathbb{C}$ admits a real Fréchet derivative at 0 . That is

$$
\left.u \mapsto \frac{d}{d t}\right|_{t=0} \Phi\left(\pi\left[\left(\exp t C_{u}^{(e)}\right) e\right]\right) \quad\left(u \in Z_{1 / 2}(e)\right.
$$

is a well-defined continuous $\mathbb{R}$-linear functional. This latter is $\mathbb{C}$-linear if and only if (19) holds.

7.2. Proposition. Let $\mathbf{U}$ be an open subset of $\mathbb{M}$. Then a function $\Phi: \mathbf{U} \rightarrow B$ into a Banach space $B$ is holomorphic if and only if for every $e \in U:=\pi^{-1} \mathbf{U}$ there exists an open subset $V$ of $Z$ with $e \in V$ along with a holomorphic function $\phi: V \rightarrow B$ such that

$$
\phi(f)=\Phi(\mathbf{f}) \quad \text { whenever } \quad f \in U \cap V .
$$

Proof. By Zorn's theorem we may assume $B=\mathbb{C}$ without loss of generality. Let $e \in U$ be arbitrary and assume the existence of a holomorphic function $\phi: V \rightarrow \mathbb{C}$ with $\phi(f)=\Phi(\mathbf{f})$, 
$(f \in U \cap V)$. Then

$$
\begin{aligned}
\left.\frac{d}{d t}\right|_{t=0} \Phi\left(\pi\left[\left(\exp t C_{i u}^{(e)}\right) e\right]\right) & =\left.\frac{d}{d t}\right|_{t=0} \phi\left(\left(\exp t C_{i u}^{(e)}\right) e\right)=\left.i \frac{d}{d t}\right|_{t=0} \phi\left(\left(\exp t C_{u}^{(e)}\right) e\right)= \\
& =\left.i \frac{d}{d t}\right|_{t=0} \Phi\left(\pi\left[\left(\exp t C_{u}^{(e)}\right) e\right]\right) \quad\left(u \in Z_{1 / 2}(e)\right)
\end{aligned}
$$

which establishes the result by Lemma 7.1.

Conversely, let $\Phi: \mathbf{U} \rightarrow \mathbb{C}$ be a holomorphic function and consider any tripotent $e \in U$. Let $\left.\Psi: Z_{(} e\right) \oplus Z_{1 / 2}(e) \rightarrow Z$ be the local chart for $\mathrm{R}$ at the point $e \in \mathrm{R}$ given by (18). Then $z \mapsto v(z):=P_{1 / 2}(e) z$ is an entire function and so is $z \mapsto(\exp D(v(z), e)) e$. Since $(\exp D(v(z), e)) e \in \mathrm{R}$ it makes sense to define $\phi: U \rightarrow \mathbb{C}$ by

$$
\phi(z):=\Phi(\pi[(\exp D(v(z), e)) e]) \quad(z \in U)
$$

where canonical map $\pi$ has been extended now to the set of von Neumann regular elements. To complete the proof we have to check that $\phi(f)=\Phi(\mathbf{f})$ whenever $f$ is a tripotent in $U$, for which it suffices to see that $[\exp D(v(f), e)] e \in \mathbf{f},(f \in \mathrm{M} \cap U)$, which is clear from the construction of $v(f)$.

7.3. Corollary. Let $X: \mathrm{M} \rightarrow \mathrm{TM}$ be an equivariant vector field such that $X(f)=P_{1 / 2}(f) h(f)$, $(f \in \mathrm{M})$, where $h: G \rightarrow Z$ is a holomorphic map defined on some open neighborhood $G$ of $\mathrm{M}$ in $Z$. Then $X$ is a holomorphic vector field on $\mathbb{M}$. With respect to the local coordinates $\widetilde{Y}^{e}$ of $\mathrm{M}$ at $\mathrm{e}$ the representation of $X$ is

$$
\left(\widetilde{Y}^{e}\right)^{\#} X:\left.u \mapsto \frac{d}{d t}\right|_{t=0}\left(\widetilde{Y}^{e}\right)^{-1}\left((\exp t X)\left(\widetilde{Y}^{e}(u)\right)\right) \quad u \in Z_{1 / 2}(e),
$$

which is a holomorphic map $Z_{1 / 2}(e) \rightarrow Z_{1 / 2}(e)$.

Proof. It suffices to see that each mapping

$$
\Phi_{e}: \mathbf{f} \mapsto\left(\widetilde{Y}^{e}\right)^{-1}([\exp t X] \mathbf{f}) \quad(e \in \mathrm{M})
$$

is holomorphic on some neighborhood $\mathbf{U}$ of the equivalence class e in $\mathbb{M}$. This is established by showing that, on some neighborhood of $e \in \mathrm{M}$, the map $\phi_{e}: f \mapsto \Phi_{e}(\mathbf{f})$ is the restriction of a $Z$-valued holomorphic map defined on some neighborhood of $e$ in $Z$.

Fix $e \in \mathrm{M}$ arbitrarily. By proposition 7.2, there exists an open neighborhood $U$ of $e$ in $Z$ along with a holomorphic function $\psi: U \rightarrow Z$ such that

$$
Y_{e}^{-1}(\mathbf{f})=\psi(f)=\psi\left(\left(\left[f+Z_{1}(f)+Z_{0}(f)\right] \cap U\right) \quad(f \in \mathrm{M} \cap U) .\right.
$$

Here $U$ is the disjoint union of the fibres $U_{f}:=\left[f+Z_{1}(f)+Z_{0}(f)\right] \cap U,(f \in U \cap \mathrm{M})$, in the sense that either $U_{f}=U_{g}$ or $U_{f} \cap U_{g}=\emptyset$, and given any couple of tripotents $f, g \in U$, we have $f \sim g \Longleftrightarrow g \in Z_{1}(f)=f+Z_{1}(f) \Longleftrightarrow g \in U_{f}$ and $U=\bigcup_{f \in U \cap M} U_{f}$. Observe that

$$
\phi_{e}(f):=\left.\frac{d}{d t}\right|_{t=0} \psi((\exp t X) f)=\psi^{\prime}(f) X(f)=\psi^{\prime}(f) P_{1 / 2}(f) h(f) \quad(f \in U \cap \mathrm{M}) .
$$

Since $\psi$ is constant along the fibres $U_{f}$, we have $\psi^{\prime}(f) \circ\left[P_{1}(f)+P_{0}(f)\right]=0,(f \in U \cap \mathrm{M})$. That is $\psi^{\prime}(f)=\psi^{\prime}(f) P_{1 / 2}(f)$ and $\phi_{e}(f)=\psi^{\prime}(f) h(f),(f \in U \cap \mathrm{M})$. Hence $\phi_{e}$ is the restriction of the holomorphic function $z \mapsto \psi^{\prime}(z) h(z),(z \in U)$, on the neighborhood $U \cap \mathrm{M}$ of $e$ in M. 


\section{References}

[1] J. AraZI - W. Kaup, On continuous Peirce decompositions, Schur multipliers and the perturbation of triple functional calculus, Math. Ann. 320, (2001) 431-461.

[2] A. Ben-Israel, T. N. E. Greville Generalized inverses Wiley, New-York 1974.

[3] C. H. CHU - J. M. Isidro, Manifolds of tripotents in JB*-triples, Math. Z. 233, (2000) 741-754.

[4] S. Dineen, P. Mellon, Holomorphic functions on symmetric Banach manifolds of compact type are constant. Math. Z. 229, 753-765 (1998).

[5] L. A. HARRIS, Bounded symmetric homogeneous domains in infinite-dimensional spaces, Proceedings on Infinite Dimensional Holomorphy, Lecture Notes in Mathematics, Vol. 364, Springer - Verlag, 1974, pp. 13-40.

[6] L. A. HARRis, W. KAUP, Linear algebraic groups in infinite dimensions, Illinois J. Math. 21, (1977) 666-674.

[7] IsIDro, J. M. - StACHÓ, L. L. On the manifold of tripotents in JB*-triples J. Math. Anal. Appl. 304 (2005) 1247-157.

[8] W. KaUp, Algebraic characterization of symmetric complex Banach manifolds Math. Ann. 228, (1977) 39-64.

[9] W. KAUP, A Riemann mapping theorem for bounded symmetric domains in complex Banach spaces Math. Z. 183 (1983) 503-529.

[10] W. KAup, On Grassmannians associated with JB*-triples, Math. Z. 236, (2001) 567-584.

[11] O. Loos, "Bounded Symmetric Domains and Jordan Pairs", Mathematical Lectures of the University of California, Irvine, 1977.

[12] M. Mackey, P. Mellon, Compact-like manifolds associated to JB*-triples Manuscripta Math. 106, (2001) 203-212.

[13] P. Mellon, Dual manifolds of JB*-triples of the form $\mathcal{C}(X, U)$ Proc. R. Irish Acad. 39A (1993) $27-42$.

[14] E. NeHER, "Grids in Jordan triple systems", Lecture Notes in Math. Vol. 1280, Springer-Verlag, Berlin - Heidelberg - New York, 1987.

[15] J. SAUTER, Randstrukturen beschränter symmetrischer Gebiete Ph. D. Dissertation, Universität Tübingen, 1995.

[16] H. UPMEIER, Symmetric Banach Manifolds and Jordan $C^{*}$-Algebras, North Holland Mathematics Studies Vol. 104, North Holland, Amsterdam 1985. 